\title{
Tsafon
}

Revue d'études juives du Nord

$72 \mid 2016$

Juifs, Israéliens, dans la littérature française et israélienne

\section{L'écriture romanesque extraordinaire en hébreu de Sayed Kashua le Peptimiste}

\section{Sâadia Agsous-Bienstein}

\section{(2) OpenEdition}

\section{Journals}

Édition électronique

URL : https://journals.openedition.org/tsafon/360

DOI : $10.4000 /$ tsafon.360

ISSN : 2609-6420

Éditeur

Association Jean-Marie Delmaire

Édition imprimée

Date de publication : 1 décembre 2016

Pagination : 47-64

ISSN : $1149-6630$

Référence électronique

Sâadia Agsous-Bienstein, « L'écriture romanesque extraordinaire en hébreu de Sayed Kashua le Peptimiste », Tsafon [En ligne], 72 | 2016, mis en ligne le 31 mai 2018, consulté le 28 juin 2021. URL http://journals.openedition.org/tsafon/360 ; DOI : https://doi.org/10.4000/tsafon.360 


\title{
L'écriture romanesque extraordinaire en hébreu de Sayed Kashua le Peptimiste
}

\author{
Sâadia Agsous-Bienstein*
}

\section{Le texte hybride palestinien en hébreu}

Sur le site internet en langue arabe, al-Masdar ${ }^{1}$, nous pouvions lire, à propos de Sayed Kashua : «le plus important écrivain hébraïque moderne est arabe $»^{2}$ (D. Kamin, 2013). Émanant d'une source arabe, de telles déclarations étaient impensables il y a encore quelques années. En effet, elles donnent à un Palestinien issu d'un village situé en Israël (Tira), un statut de «star » d'une littérature censée être représentative de la majorité juive.

Sayed Kashua appartient au cercle très restreint d'écrivains issus de la minorité palestinienne en Israël ${ }^{3}$ qui produisent des romans en hébreu. Défini comme texte hybride (Suleiman, 2013), cette écriture romanesque

\footnotetext{
* Centre européen de sociologie et science politique (CESSP-EHESS) et Centre de recherche français à Jérusalem (CRFJ).

L'intitulé de l'article fait allusion au titre du roman de l'écrivain palestinien Émile Habibi (1922-1996) Les aventures extraordinaires de Saïd le Peptimiste.

Les références entre parenthèses renvoient à la bibliographie en fin d'article. Le signe suivant (*) qui suit certaines citations indique au lecteur que ces dernières sont traduites par nous-même de l'anglais, de l'arabe et de l'hébreu.

${ }^{1}$ http://www.al-masdar.net/

${ }^{2}$ Issu d'une famille palestinienne musulmane, Kashua est né en 1975 à Tira. À peine âgé de 15 ans, il obtient une bourse pour un lycée juif prestigieux de Jérusalem (Jerusalem School of Science and Art) qui le met ainsi très tôt en contact avec la langue hébraïque. C'est au lycée qu'il apprend à manier cette langue au détriment de l'arabe et c'est à l'Université Hébraïque de Jérusalem qu'il étudie la sociologie et la philosophie. Sayed Kashua est régulièrement invité à des salons de livres internationaux et est lauréat de plusieurs prix.

${ }^{3}$ Nous les nommerons tout au long de cet article Palestiniens en Israël.
} 
combine l'identité arabe palestinienne de l'écrivain et son écriture hébraïque. Pour Yassir Suleiman :

[...] Les textes hybrides (prose-fiction) sont écrits par des auteurs d'origine arabe - par descendance ou par héritage -, mais dans une autre langue comme le français, l'anglais ou l'hébreu en ce qui concerne les Palestiniens en Israël. Ces textes sont distribués dans différents lieux de culture qui pourraient être définis selon la langue dominante du texte, l'origine de l'auteur ou dans un tiers espace, ou dans une zone floue située entre ces deux mondes.* (Y. Suleiman, $2013: 167$ )

Le roman palestinien en hébreu a pris racine dès 1966 avec la publication par Atallah Mansour (1935-) de באור חדש (Sous un nouveau jour $)^{4}$. Il s'agit d'une production romanesque certes limitée, mais qui projette sur la scène littéraire hébraïque et arabe la problématique de l'identité du Palestinien en Israël. Les personnages romanesques ont la particularité de rebondir sur la scène politique et de discuter le statut social et politique du Palestinien au sein d'une hégémonie juive. Sayed Kashua appartient à la troisième génération de la Nakba (catastrophe) ${ }^{5}$ (D. Halutz, 2010), et l'identité palestinienne dans sa relation problématique avec l'israélienne demeure le centre névralgique de son écriture inscrite dans le $\mathrm{XXI}^{\mathrm{e}}$ siècle.

Pour les écrivains palestiniens bilingues, l'identité modelée à partir d'un changement brusque de statut de leur groupe social ne pouvait se forger sans la prise en compte de cet «autre» et de sa langue. Plus encore, elle a inscrit cet « autre » dans son sillon identitaire. Cet « autre » est multiple : il est Juif, il est Palestinien en exil et en Israël, il est hébreu et arabe et il est à l'intérieur et à l'extérieur. Il est avant tout bilingue, et présent dans le domaine de la traduction, de la poésie, du théâtre et de la prose. Les auteurs bilingues tels que Atallah Mansour, Anton Shammas et Naïm Araïdi exploitent leurs statuts (en tant que membres de la minorité), le transforment en source de création littéraire pour ainsi favoriser l'émergence d'un espace de négociation.

Quelques notions de Deleuze sur les problématiques liées au bilinguisme nous interpellent. Deleuze explique que «le multilinguisme n'est pas seulement la possession de plusieurs systèmes dont chacun serait homogène en lui-même; c'est d'abord la ligne de fuite ou de

\footnotetext{
${ }^{4}$ Pour les écrivains bilingues arabe-hébreu nous citerons : Anton Shammas (1950-), Naïm Araïdi (1950-2015), Salman Masalha (1953-) et Aymen Sickseck (1984-).

${ }^{5}$ Les Palestiniens utilisent le terme Nakba pour désigner leur défaite en 1948.
} 
variation qui affecte chaque système en l'empêchant d'être homogène » (G. Deleuze \& C. Parnet, 1996 : 10-11).

Ainsi, Sayed Kashua, qui a fait l'usage de l'hébreu pour la création littéraire, n'est pas, par son multilinguisme, un détenteur de plusieurs systèmes. Cependant, ses romans représentent une «ligne de fuite» et une « variation » face à la littérature hébraïque. Cette « ligne de fuite » et cette « variation » ont, pour ainsi dire, ébranlé cette littérature. En effet, l'usage même de l'hébreu par des non-Juifs pour décrire et narrer une réalité liée à la situation des individus membres de la minorité palestinienne en Israël est considéré comme une remise en cause du facteur juif, élément fondateur de la littérature hébraïque. De ce fait, Kashua a explicité l'existence d'une autre possibilité, d'un autre lieu capable de filtrer à partir de deux points antagonistes et de reconstruire l'image du Palestinien dans la littérature hébraïque.

Les écrivains palestiniens ont eu recours au bilinguisme littéraire ${ }^{6}$ jusqu'aux années 1990. Néanmoins, ceux qui publient des romans en hébreu à partir des années 2000, tel Sayed Kashua, le font exclusivement dans cette langue. Si Kashua ne pratique pas le bilinguisme littéraire, alors que ses langues sont doubles, comment le situer ? Pour répondre à la problématique des auteurs qui ne sont pas à proprement parler des écrivains bilingues dans leur écriture, mais le sont par leur statut d'immigrés ou parce qu'ils sont issus de l'immigration postcoloniale, Rainier Grutman ${ }^{7}$ suggère la notion « translingues » :

En insistant sur le sens étymologique de la préposition latine : « de l'autre côté, au-delà, par-delà, par-dessus ». Il est dommage, mais symptomatique que tant de mots français commençant par trans (comme «transfuges » ou «transplantés »), aient une connotation négative. En latin, la préposition était suivie de l'accusatif parce qu'elle exprimait un mouvement, un déplacement dans l'espace (transferre), nuance qui convient parfaitement aux écrivains translingues, chez qui s'est opéré un véritable transfert linguistique. Ils ont laissé derrière eux un pays, une culture et une langue et ont dû s'adapter (linguistiquement et culturellement) à la société d'accueil. (A. Gasquet \& M. Suãrez, 2007 : 39)

Le terme «translingue » est approprié à Sayed Kashua qui n'a pas laissé derrière lui un pays et une culture, mais une langue et une littérature (arabe) pour s'adapter à une langue et à une littérature d'accueil (hébraïque) qui est devenue sienne. C'est fondamental pour

\footnotetext{
${ }^{6}$ Par bilinguisme littéraire, nous entendons l'acte d'écrire de la littérature en langue arabe littéraire et en langue hébraïque.

${ }^{7}$ Université d'Ottawa.
} 
comprendre la contradiction qui s'opère dans l'identité palestinienne présente dans son écriture romanesque. Néanmoins, notons que Kashua a pratiqué son bilinguisme dans l'écriture du scénario de la série télévisée Avoda Aravit (Travail arabe) puisqu'une majorité des dialogues sont écrits en dialecte palestinien, langue vernaculaire de la minorité palestinienne.

Ce qui importe est la priorité accordée non pas au bilinguisme littéraire, mais à l'œuvre. En d'autres termes, comprendre le choix de la littérature pour exprimer un espace identitaire dans une œuvre proposée comme « ligne de fuite ». Le texte palestinien hybride adhère à la notion de la littérature mineure ${ }^{8}$ et Sayed Kashua a fait le choix de la littérature pour «trouver son propre point de sous-développement, son propre patois, son tiers monde à soi, son désert à soi ». (G. Deleuze \& Guattari, $1975: 33)$.

\section{Remarques sur les transformations génériques dans les textes hybrides de Sayed Kashua}

Sayed Kashua puise, pour ses écrits littéraires, à partir d'événements historiques et politiques qui coïncident avec ses mémoires d'enfance et de vie adulte partagées entre un espace villageois, Tira, et une ville, Jérusalem. Il y anime des personnages palestiniens et juifs en mouvement dans des intrigues présentées par des narrateurs, parfois autodiégétiques et d'autres fois hétérodiégétiques, qui combinent un mode narratif omniscient et interne.

Son premier roman ערבים רוקדים (Les Arabes dansent aussi) est inscrit dans le registre de l'autofiction et est publié en 2002 au cœur de la seconde Intifada. Son auteur, alors journaliste indépendant pour l'hebdomadaire Haïr, conçoit son œuvre au cœur d'une révolte collective qu'il a détournée pour énoncer une rébellion individuelle. C'est une réussite sans précédent pour un écrivain palestinien d'expression hébraïque. Son roman est lu en Israël et traduit en plusieurs langues et l'auteur est lauréat de plusieurs prix israéliens et internationaux. Ce

\footnotetext{
${ }^{8}$ Pour notre thèse de doctorat, nous avons étudié les problématiques des langues et des identités à partir de ce principe : l'usage d'une minorité, c'est-à-dire les Palestiniens (locuteurs arabes), de la langue majeure, c'est-à-dire l'hébreu, (langue de la majorité juive en Israël). En d'autres termes, nous avons posé la question suivante : que font les écrivains palestiniens, Atallah Mansour, Anton Shammas et Sayed Kashua, de la langue hébraïque dans leurs romans?
} 
triomphe est un encouragement pour la poursuite dans son écriture littéraire en hébreu.

Le héros-narrateur autodiégétique n'a pas de nom comme la plupart des personnages membres de sa famille. Cependant son «je» est signifiant et projette un héros décalé, sans qualité, mis à l'écart par les Juifs israéliens et aliéné par les siens. C'est le Palestinien d'Israël qui danse contre une société palestinienne restrictive et une société israélienne exclusive. Les autres personnages, Palestiniens des Territoires et Juifs-israéliens, sont souvent nommés et situés selon leurs espaces géographiques partagés entre l'État d'Israël et les Territoires occupés.

Avec la publication de ויהיה בוקר (Et il y eut un matin), Sayed Kashua réitère sa volonté d'adhérer au cercle de la littérature hébraïque ${ }^{9}$ et se distingue d'Atallah Mansour et d'Anton Shammas par sa constance dans la production de textes littéraires en hébreu. Pour son deuxième roman, publié en 2004, l'auteur s'est écarté du vécu individuel pour narrer le collectif minoritaire dans un espace situé entre village et ville, mais qui demeure anonyme. " Ce sont des gens normaux qui vivent dans une situation qui ne l'est pas » (Kashua, 2008). C'est ainsi que l'auteur décrit ses personnages, qui sont dans leur majorité Palestiniens.

Le narrateur-personnage autodiégétique n'a pas de nom, il en sera de même pour les autres membres de sa famille. Son récit est fait en focalisation interne. L'intrigue, unique, et sa résolution sont suggérées à travers le regard et les ressentis du personnage principal. Il est originaire d'un village palestinien situé en Israël, frontalier avec la Cisjordanie et il travaille en ville. Contrairement au premier roman, les lieux ne sont pas nommés. De nouveau, le contexte est celui de la seconde Intifada et plus particulièrement de ses répercussions politiques sur la situation fragile de la minorité palestinienne d'Israël. Le choix d'aborder l'identité minoritaire dans son aspect collectif octroie à Et il y eut un matin une dimension politique plus importante que celle du premier roman.

Son dernier roman גוף שני יחיז (La deuxième personne) publié en 2010, occupe à nouveau l'espace de Jérusalem et la période de la deuxième Intifada. Il plonge dans un genre réaliste l'identité de deux personnages palestiniens face à l'identité juive majoritaire. Sayed Kashua

\footnotetext{
${ }^{9}$ Lors du Salon du livre qui eut lieu à Paris en 2008, Kashua a été présenté comme une vedette par les représentants culturels israéliens du fait qu'il soit Palestinien doté d'une écriture littéraire en langue hébraïque. Kashua a également exprimé dans le documentaire qui lui a été consacré par Dorit Zimbalist «Sayed Kashua - Forever Scrared » son souhait de faire partie de la littérature hébraïque.
} 
fait chevaucher deux récits : le premier narre, à la troisième personne, le tourment d'un avocat, Palestinien d'Israël sans nom, qui découvre une éventuelle relation extra-conjugale qu'entretiendrait sa femme avec un Juif-israélien nommé Yonatan. Le second raconte à la première personne la vie d'Amir Lahab, également membre de la minorité palestinienne d'Israël, et le processus d'usurpation de l'identité légale d'un Juifisraélien nommé Yonatan.

Coexistent, dans cette intrigue complexe, deux personnages qui se rencontrent à la fin du roman, deux narrateurs : hétérodiégétique dans le récit du personnage principal l'avocat et autodiégétique pour le récit d'Amir Lahav. Le point de vue narratif est omniscient pour les deux, seulement le narrateur du récit de l'avocat bénéficie d'une focalisation totale puisqu'il maîtrise également le récit d'Amir Lahav.

Ce sont les lignes principales à partir desquelles Sayed Kashua a narré le Palestinien d'Israël avec l'ironie et l'humour qui lui sont particuliers.

\section{Sayed Kashua : le Peptimisme comme stratégie littéraire}

Kashua combine humour, ironie et parfois dérision, pour raconter des histoires diverses. Elles se présentent parfois sous un aspect optimiste ou pessimiste. Il illustre parfaitement la notion «peptimiste » créée par Émile Habibi (É. Habibi, 1987) pour le personnage principal de son roman Les aventures extraordinaires de Saïd le Peptimiste. Saïd dit :

Tenez, moi, par exemple : eh bien, je ne fais pas de différence entre l'optimisme et le pessimisme, et je me demande bien lequel des deux me caractérise le mieux. Le matin, quand je me réveille, je remercie Dieu de ne pas m'avoir fait périr pendant la nuit. Si, dans la journée, il m'arrive quelque chose de désagréable, je le remercie de ce qu'il ne me soit rien arrivé de pire. Que suis-je donc, optimiste ou pessimiste? (É. Habibi, $1987: 23$ )

Saïd représente une métaphore, celle de cette minorité palestinienne née en 1948 avec le nouvel État d'Israël. Une minorité satisfaite d'avoir pu rester sur ses terres et satisfaite de n'avoir pas connu pire situation que l'expulsion. Précisément à l'instar de Saïd, Kashua écrit d'une part : "J'aurais aimé pouvoir être fier d'être arabe israélien, mais comment puis-je le faire si je ne suis pas vraiment reconnu comme citoyen à part entière ${ }^{*}$ (D. Berrin, 2012). D'autre part, il reste optimiste grâce aux soutiens d'écrivains juifs israéliens tels qu'Amos Oz et Etgar 
Keret qui l'encouragent à persévérer dans l'écriture en hébreu. Ce mélange entre positif et négatif, société palestinienne et israélienne, arabe et hébreu, le tout teinté d'humour et d'ironie est devenu central dans l'œuvre de Sayed Kashua et l'identité est son obsession.

Être Palestinien et écrire en hébreu sur les Palestiniens n'est pas le seul critère pour une reconnaissance sur le plan littéraire. Kashua explique que : «s'il y a un autre jeune Palestinien qui écrit en hébreu, je n'aurai plus de travail [...] je suis le seul qui profite du racisme dans ce pays [...] et c'est grâce à lui que je gagne ma vie »* (A. Neslen, 2011 : 103). Ainsi, l'écrivain exploite la combinaison paradoxale (palestinien langue hébraïque) pour vendre des livres et pour transmettre la rude réalité au lecteur. Il fait le choix d'écrire avec humour sur son intimité. Il illustre bien le propos de Frye qui explique que c'est : « le comique qui concilie le héros avec la société » (Todorov, 1970 : 16). C'est la stratégie narrative de Kashua. Autrement dit, son conflit identitaire est devenu source de création, et l'humour une véritable stratégie de survie face au lecteur.

Arthur Neslen pense que Kashua doit sa carrière au racisme juif et parfois à l'antiracisme (A. Neslen, 2011), alors que pour Yanoshevsky il est question d'imposture identitaire car Kashua exprime un processus complexe d'identification entre le regard minoritaire et le désir d'assimilation à la majorité (G. Yanoshevsky, 2011). L'imposture devient alors une posture :

[...] il s'agit plutôt de la manière dont Kashua fabrique, à travers ses romans et ses chroniques, le personnage littéraire et journalistique d'un Arabe qui se dépeint constamment en Juif, un spécialiste de l'art d'endosser une fausse identité - tant et si bien que même les Arabes le prennent pour un Juif -, mais dont la « vraie identité » est susceptible d'être révélée à tout moment. (G. Yanoshevsky, 2011:58)

Dans les récits littéraires de Sayed Kashua, l'ironie se déploie comme une manœuvre et donne à l'auteur liberté ${ }^{10}$ et franchise pour raconter une situation difficile, pénible, sans pour autant perturber son

\footnotetext{
${ }^{10}$ Pierre Schoentjes parle d'ironie moderne pour la différencier de celle des romantiques "Chez les différents auteurs qui abordent l'ironie moderne à travers la production littéraire, l'ironie apparaît comme une forme de liberté : liberté de l'auteur de tenir ses personnages à distance, manière de se libérer du poids des sentiments, manière aussi de prendre ses distances avec les conventions de la société. Cette association de l'ironie et de la liberté sera plus forte encore chez ceux qui observent l'ironie non plus dans les œuvres, mais dans le monde qui les entoure » (P. Schoentjes, $2001: 259$ ).
} 
statut dans la littérature hébraïque. Dans son commentaire sur l'ironie moderne Pierre Schoentjes explique que: «l'ironie n'est pas cette grossière plaisanterie railleuse qui dit le oui pour le non mais bien un point de vue que l'auteur choisit pour observer la vie, et qui se caractérise par l'absence de jugement porté sur les personnages » (P. Schoentjes, 2001 : 258). C'est de cela dont il est question dans les romans de Sayed Kashua. L'hébreu, combiné à l'ironie, est pour lui le moyen de mettre en œuvre un rapport dialogique avec le lecteur juif israélien. Gustave Khan précise que : «le romancier ironiste, penchera plutôt à instruire ses lecteurs » (P. Schoentjes, 2001: 258), de la même manière, Sayed Kashua est ironiste et il a comme objectif d'instruire le lecteur hébraïque.

Les textes hybrides de Sayed Kashua imposent trois pistes principales : d'abord l'identité d'un membre de la minorité palestinienne représente l'épicentre des récits. Ensuite ses écrits sont ancrés dans le $\mathrm{XXI}^{\mathrm{e}}$ siècle, car la deuxième Intifada est la référence temporelle qui s'impose. Enfin, il existe un changement dans le genre et le registre d'écriture. En effet, après avoir expérimenté l'autofiction, Kashua s'est engagé dans le registre fantastique avant de composer son dernier roman dans le réalisme, propre à ses prédécesseurs. Inconnu est l'engagement de Sayed Kashua dans le registre fantastique, précisément à travers son unique nouvelle, (Herzl disparaît à minuit), publiée en 2005 dans le quotidien israélien Haaretz dans le cadre de ses chroniques journalistiques.

\section{Cendrillon à Jérusalem, une nouvelle palestinienne en hébreu}

\section{Palestinien et Juif-israélien dans le fantastique}

L'unique nouvelle de Kashua se lit comme un récit fantastique. Contrairement aux précédents romans, le narrateur est hétérodiégétique et le personnage principal est identifié par le nom Herzl Hliwa. À moitié juif et à moitié arabe, ce nom combine une référence au fondateur du sionisme, Theodore Herzl, et une référence arabe, Hliwa, qui provient probablement de la racine $h l w$ synonyme de beauté et de friandises, mais qui ne renvoient à une aucune figure publique arabe notoire. Puisque le nom est double, ainsi est l'espace dans lequel Kashua a choisi de narrer le périple et l'aventure d'Herzl Hliwa : c'est Jérusalem, un espace ancré entre sa partie arabe (orientale) et sa partie juive (occidentale). Ce lieu intervient pour donner une réalité à l'aventure fantastique de ce personnage hors normes. 
Un an avant la guerre ${ }^{11}$, une femme, stérile, la quarantaine passée, est face au Mur occidental. Elle supplie Dieu de lui donner un enfant même à moitié arabe. Le rêve devient réalité et bonheur. Puis la vie d'Herzl Hliwa devient un enfer. Le jour, il est Juif et parle hébreu et à partir de minuit, il devient Palestinien et parle uniquement l'arabe : « il ne devient pas n'importe quel Arabe, mais un fier nationaliste qui refuse de se rendre dans la ville occidentale, car il ne veut pas souffrir l'humiliation »* (S. Kashua, 2005).

Herzl Hliwa réside à Jérusalem-Ouest et entretient une relation amoureuse avec Noga, cependant il n'ose pas lui avouer la vérité sur sa double identité. Suite aux pressions de son amoureuse, il décide de l'embarquer dans son périple pour lui révéler sa transformation identitaire palestinienne nocturne ainsi que la transformation identitaire juive matinale. Noga découvre un amant qui lui est étranger.

T. Todorov écrit que «le fantastique, c'est l'hésitation éprouvée par un être qui ne connaît que les lois naturelles, face à un événement en apparence surnaturel» $(\mathrm{T} \text {. Todorov, } 1970: 29)^{12}$. C'est en rapport avec cette définition que la nouvelle de Sayed Kashua est inscrite dans ce registre.

La situation initiale de l'intrigue se présente, dès la scène d'exposition, «Herzl Hliwa pousse un cri et secoue brusquement sa tête avec horreur. Il reprend conscience très vite - c'est déjà arrivé auparavant $-[\ldots] »^{*}(\mathrm{~S}$. Kashua, 2005). Les péripéties et le dénouement se poursuivent, cependant Herzl devient personnage principal et Hliwa narrateur hétérodiégétique qui fait le récit de sa moitié. L'identité dédoublée surnaturelle d'Herzl Hliwa plante le récit dans le genre fantastique. Sayed Kashua a certes fait usage de référents historiques en lien avec la réalité du lecteur (la guerre), linguistiques (arabe et hébreu), spatiaux (la ville de Jérusalem) et politiques (le conflit israélopalestinien). Cependant, l'intrusion de ce personnage qui se métamorphose de façon surnaturelle provoque « une incertitude » chez le lecteur. On se préoccupera donc du rôle du narrateur et personnage

\footnotetext{
${ }^{11}$ Le contexte de la guerre qui n'est pas spécifié. Il est probablement question de la période post-1967 puisque le Mur occidental était sous contrôle jordanien de 1948 à 1967, pour passer ensuite sous contrôle israélien.

${ }^{12}$ Éric Bordas \& all offrent une définition similaire : «Le fantastique ne se confond donc pas avec l'affabulation des féeries, mais se caractérise par une intrusion brutale du mystère dans la vie réelle. » (E. Bordas \& all., $2009:$ 176).
} 
principal dans l'espace de Jérusalem pour démontrer cette hésitation et sa transférabilité entre lecteur et personnage ${ }^{13}$.

\section{Cendrillon ${ }^{14}$ à Jérusalem}

Kashua a choisi la ville de Jérusalem pour raconter le périple d'Herzl. C'est un espace ancré entre sa partie arabe (orientale) et sa partie juive (occidentale). Herzl se sent menacé à Jérusalem-Est et Hliwa considère Jérusalem-Ouest comme un symbole colonial. Ce lieu intervient pour donner une réalité à ce personnage fantastique, mais sa dichotomie est exprimée dans les caractéristiques de deux composantes.

Parce que la métamorphose palestinienne d'Herzl se produit, exactement comme dans le conte de Cendrillon, à minuit, cela l'a empêché de passer avec son amante un seul moment intime. Il n'a pas le courage de lui avouer qu'à minuit il devient Hliwa, parle l'arabe, rejoint les cercles de militants palestiniens à Jérusalem-Est et couche avec d'autres femmes, des volontaires internationales. En définitive, il l'invite à un voyage pour être le deuxième témoin, hormis sa mère, de sa transformation identitaire palestinienne nocturne. Noga découvre un amant qui, à partir de minuit, ne comprend pas l'hébreu, fume et remplace le vin par l'arak ${ }^{15}$.

En revanche, le personnage semble bien maîtriser ce dédoublement. $\mathrm{Au}$ retour de son aventure palestinienne dans un bar situé à Sheikh Jarrah $^{16}$, il ouvre une armoire fermée à clef qui contient des livres en arabe. Il en saisit un et lit jusqu'à épuisement :

« Que va-t-il se passer avec toute cette histoire arabe?» « Si tu veux mon avis », répond-il en se dirigeant vers les toilettes «ils peuvent tous brûler en enfer ».* (S. Kashua, 2005)

\footnotetext{
${ }^{13}$ En effet la définition du fantastique de Tzvetan Todorov souligne cette hésitation partagée entre lecteur et personnage : « D'abord, il faut que le texte oblige le lecteur à considérer le monde des personnages comme un monde de personnes vivantes et à hésiter entre une explication naturelle et une explication surnaturelle des événements évoqués. Ensuite, cette hésitation peut être ressentie également par un personnage ; ainsi le rôle de lecteur est pour ainsi dire confié à un personnage et dans le même temps 1'hésitation se trouve représentée, elle devient un des thèmes de l'œuvre $[\ldots]$ » $\mathrm{T}$. Todorov, $1970: 39$ ).

${ }^{14}$ La traduction du titre de la nouvelle en anglais est Cendrilla. Pour la traduction en français, nous avons opté pour Herzl disparaît à minuit. Nous souhaitions rester fidèle au titre en hébreu qui projette le mystère et le doute.

${ }^{15}$ Boisson alcoolisée produite et consommée dans la région du Moyen-Orient.

${ }^{16}$ Quartier palestinien de Jérusalem-Est.
} 
Cette nouvelle est censée aborder les difficultés qu'affrontent Herzl Hliwa pour combiner les deux identités accordées par le Divin dans une situation politique très tendue. Il n'existe aucun moment où langue arabe et hébraïque ou bien l'identité palestinienne et juive se croisent. Lorsque le personnage est Hliwa, il ignore l'hébreu, et lorsqu'il est Herzl il ignore l'arabe. Ils partagent le même corps, mais s'ignorent sauf à l'incipit.

Contrairement à ce à quoi Kashua nous a habitués, le personnage palestinien, dans cette nouvelle, n'aspire pas à être Juif. Mais il crée une identité juive en son sein et la justifie par une volonté divine. Il est possible de lire la nouvelle comme une solution envisageable du partage d'une terre entre Juifs et Palestiniens. Néanmoins, elle se représente d'abord comme un théorème de la connaissance de l'auteur, Kashua, de ce Juif israélien, puisqu'il accède à sa propre intimité. Plus encore, il n'est plus question, dans ce court récit, de l'identité palestinienne, dans sa variante individuelle et collective, mais il s'agit bien de la problématique de l'identité du Juif israélien face à l'identité palestinienne.

\section{Jeu de la fragmentation des identités}

Le titre Herzl disparaît à minuit pose immédiatement la question suivante : si Herzl disparaît, où est Hliwa ? Il n'est pas le personnage principal, mais le narrateur omniscient. Son rôle est d'exposer le récit d'Herzl, personnage représentatif des Juifs israéliens. En accordant la première place à ce Juif, Kashua expose ainsi la relation majoritéminorité à partir du regard d'Herzl et non de celui de Hliwa. Contrairement aux premiers romans de Kashua, ce n'est plus le Palestinien qui est gêné par son identité double (palestinienne israélienne), mais c'est le Juif qui ne s'accepte pas dans la peau d'un Arabe. Témoin cet extrait dans lequel Herzl quitte l'hôtel de JérusalemEst pour se rendre chez lui à Jérusalem-Ouest :

Il n'a aucune idée de l'endroit où il va, alors que la nuit dernière il était un autochtone expérimenté accompagnant l'invitée allemande du bar de Sheikh Jarrah à l'auberge située dans la vieille ville. Il tente sa chance, se rappelant qu'il doit marcher vers l'ouest, loin du lever du soleil [... $]^{*}$ (S. Kashua, 2005)

Dès son réveil dans une chambre d'hôtel du quartier de JérusalemEst, il a peur, il se dépêche de s'habiller, accélère ses pas dans la ville orientale. Le narrateur fait son récit en suivant les battements de son cœur. Son récit s'accélère lorsque Herzl se sent en danger dans la ville 
orientale. De plus, nous sommes vendredi, jour de la prière des musulmans. Il ralentit lorsqu'il retrouve son calme à la vue de soldats patrouilleurs israéliens. Herzl est enfin rassuré lorsqu'il arrive dans sa rue. Kashua nous fait ainsi vivre la course d'Herzl qui évite l'Orient, le Palestinien, et se rapproche de son Occident, loin des brouhahas du vendredi matin.

Le supposé personnage principal de cette nouvelle porte un nom double. Cependant, Hliwa n'apparaît qu'une seule fois, à l'incipit. Le nom, bien qu'hybride (juif-palestinien), est consciemment dissocié dans le récit pour décrire la fragmentation soutenue d'un point de vue identitaire, comme un jeu auquel l'auteur a participé. « Jeu » est un motclé puisque Noga est convaincue que son amant joue à un jeu lorsqu'il lui déclare être à moitié Arabe et l'invite à être témoin de son arabité nocturne :

Noga commence à être fatiguée de ce jeu. Elle supplie Herzl d'arrêter. Ils vont enfin partager une nuit entière et il continue à jouer à ce jeu idiot. Herzl ne comprend pas un seul mot de ce qu'elle dit et tente de parler en anglais, mais Noga se sent un peu idiote et refuse de se prêter à ce jeu.* (S. Kashua, 2005)

Ainsi, Sayed Kashua s'est livré à un jeu dans lequel le patronyme juif-palestinien est situé dans le texte tel un jeu de cartes. Par l'utilisation de cette carte hybride, l'auteur nous engage immédiatement dans la problématique de la nouvelle qui consiste en une supposée impossibilité identitaire dédoublée.

Le nom Herzl isolé apparaît dix fois comme une suite de ce jeu entamé avec Herzl Hliwa. Lorsque le personnage transparaît dans sa peau palestinienne, il n'est pas appelé Hliwa, mais Herzl. Lorsqu'il parle l'arabe et lorsqu'il insulte les soldats israéliens, lorsqu'il promène Noga dans Jérusalem-Est, il est Herzl. Mais lorsque le personnage redevient juif et vit dans son quartier de Jérusalem-Ouest, il se nomme Hliwa. Ainsi Kashua a choisi de brouiller les cartes pour exprimer cette identité hybride hors du commun, voire fantastique.

Dans Herzl disparaît à minuit, c'est l'identité d'Herzl qui est importante dans sa relation avec la palestinienne, qui n'est pas inscrite dans une identité supposée supérieure ou inférieure. C'est le contexte qui met des pressions sur Herzl par rapport à Hliwa. Il s'agit en fin de compte d'une nouvelle qui aborde le psychique du Juif israélien face à son alter-ego le Palestinien. La chute du récit est symbolisée par l'armoire remplie de livres en arabe. Cette armoire fermée à clé est 
encore une fois une allégorie de cette identité palestinienne que le Juif israélien cache, refuse et rejette. C'est le malheur d'Herzl. L'auteur aurait donc pu titrer son récit « Hliwa disparaît ».

En tant que lecteurs, nous hésitons sur la véracité de l'authenticité identitaire d'Herzl-Hliwa. Cette hésitation est transférée au personnage principal Herzl qui doute de son autre moitié, palestinienne, qui se manifeste le soir, loin des regards curieux et hostiles. Cependant, Hliwa, ce narrateur omniscient, tente d'effacer la distance existant entre le réel et l'irréel pour qu'Herzl n'hésite plus.

Herzl Hliwa, ce nom « moitié-moitié », est en soi la représentation d'un No Man's land ${ }^{17}$. Lieu où il est question d'un nom dont il est impossible de distinguer les composantes, entre patronyme et prénom, où il est question d'une manifestation identitaire dédoublée lors de la seconde Intifada. Et ce lieu devient un refuge indépendamment des deux récits, pour le Palestinien et l'Israélien.

Batya Shimony explique que la division totale de ces deux identités offre le choix au personnage entre une identité «blanche» dominante ou une affiliation à l'identité palestinienne (B. Shimony, 2013). Cependant, par ce récit, Sayed Kashua exprime la possibilité d'un espace hybride, d'un tiers-espace selon la notion d'Homi Bhabha :

[...] si l'hybridité est importante, ce n'est pas qu'elle permettrait de retrouver deux moments originels à partir desquels un troisième moment émergerait, l'hybridité est plutôt pour moi le « Tiers-espace » qui rend possible l'émergence d'autres positions. Ce Tiers-espace vient perturber les histoires qui le constituent et établit de nouvelles structures d'autorité, de nouvelles initiatives politiques, qui échappent au sens commun. (H. Bhabha, 2007)

L'espace hybride romanesque conçu par Kashua prend forme à partir des lisières de deux récits, mais qui semble hors de portée et isolé dans la situation actuelle. L'auteur a révélé à travers ses deux premiers romans son écriture sur l'identité palestinienne: elle est impossible lorsqu'elle relève de l'individuel et incertaine lorsqu'elle porte sur le

\footnotetext{
${ }^{17}$ Si l'expression anglaise " No-Man's-Land » représente un espace non habité par des humains, il est dans le contexte israélien un symbole spatial, social, identitaire, culturel et politique qui échappe aux normes hégémoniques. À titre d'exemple l'ouvrage de Lital Levy (2014) Poetic Trespass: writing between Hebrew and Arabic in Israel/Palestine est une narration sophistiquée des possibilités de production littérature, artistique et cinématographique dans le contexte d'un long conflit politique par des membres de groupes minoritaires, Palestiniens et Mizrahim (Juifs-Arabes). C'est un NoMan's Land, un espace dans lequel Palestiniens-Juifs et Arabe-Hébreu se rencontrent par l'intermédiaire de la littérature et la traduction.
} 
collectif. Cette nouvelle atteste également de sa grande virtuosité - voire de son génie - à écrire sur l'identité juive. Ainsi, ce court récit se propose comme une expérimentation de l'auteur sur ses lecteurs du genre fantastique. Cela lui a permis de changer l'orientation générique de son dernier roman et de créer des personnages juifs-israéliens plus élaborés.

\section{Redéfinir les identités par l'écriture littéraire}

Sayed Kashua, l'unique représentant du roman palestinien en hébreu au XXI ${ }^{\mathrm{e}}$ siècle, décrit dans la langue de « l'autre » le Juif-israélien et à partir d'un espace situé dans l'entre-deux des identités multiples, impossibles, incertaines et probables. Des identités partagées entre optimisme et pessimisme, deux pôles contradictoires qui marquent et traduisent la fragilité et la sensibilité des membres d'une minorité à laquelle il appartient. Kashua a conçu sa propre patrie, celle de l'écriture romanesque en hébreu. Née certes à partir d'une appartenance minoritaire et d'une identification avec la majorité, mais c'est une écriture développée à partir des lisières de ces deux pôles de la société israélienne auxquelles il refuse de se soumettre «Je refuse la sacralisation de l'identité arabe comme de l'identité juive » (S. Kashua : 2011).

Raconter la vie dans cet entre-deux en hébreu, relève d'une stratégie d' "infiltration du contexte culturel hébraïque » (H. Hever, 2001), plutôt que d'un choix linguistique, qu'il a enrichi d'ironie, de fantastique et de réel. L'imbrication entre l'écrivain, le politique et le collectif, évoquée par les théoriciens de la littérature mineure, nous offre les moyens de considérer l'écriture littéraire en hébreu de Kashua comme le moyen d'expression d'une autre possibilité, d'une autre identité qui se situe sur les lisières du Majeur et du Mineur :

[...] c'est la littérature qui produit une solidarité active, malgré le scepticisme ; et si l'écrivain est en marge ou à l'écart de sa communauté fragile, cette situation le met d'autant plus en mesure d'exprimer une autre communauté potentielle, de forger les moyens d'une autre conscience et d'une autre sensibilité. (Deleuze \& Guattari, $1975: 31-32$ )

C'est cette sensibilité que Kashua a exprimée par la littérature. Ses romans décrivent des identités palestiniennes particulières aux membres de la minorité palestinienne d'Israël; des identités qui empruntent un sentier impossible et incertain. Elles sont aussi déchirées, instables, et perturbées lorsqu'elles se définissent à partir de cette relation 
conflictuelle entretenue avec le Juif israélien, une relation qui se situe entre familiarité et inaccessibilité.

À cause de la situation politique, il est impossible pour Kashua de ne pas écrire, il lui est impossible d'écrire un roman non politisé et il lui est également impossible de ne pas être peptimiste. C'est un peptimisme marqué par la relation entretenue avec "l'autre » familier et étranger et par les relations entre minorité et majorité. C'est à partir du Palestinien et du Juif israélien que Kashua a choisi de marquer son écriture littéraire. Une écriture qui perturbe les bases mêmes de l'identité moderne palestinienne et israélienne.

Yassir Suleiman explique que dans le domaine linguistique le texte hybride possède une nature dialogique "dans le sens que la langue manifeste/présente du texte suggère toujours sa langue cachée/absente » (Y. Suleiman, 2013 : 167). Ainsi, cette nouvelle (et ses romans) cache en premier le lien fort qu'entretient l'auteur avec l'identité qui s'articule à partir de son espace géographique et historique palestinien. En d'autres termes, seuls la littérature et ses procédés génériques ont permis à Kashua d'exprimer son attachement à sa terre et à son identité palestiniennes. Elle a également permis d'insérer au sein de la littérature hébraïque l'image d'un personnage palestinien modifiée par des écrivains qui brisent l'homogénéité juive sur cette littérature. Par le choix de la langue et de la problématique identitaire, combinées au genre fantastique, Kashua a œuvré pour établir un dialogue avec le lecteur israélien (juif). Kashua a quitté Israël pour une autre vie aux États-Unis, il a déclaré avant son départ: «Je voulais raconter aux Israéliens une histoire, l'histoire palestinienne » (S. Kashua, 2014). 


\section{Bibliographie}

- Berrin D. (2012). «Fish out of water: An evening with Arab-Israeli writer Sayed Kashua », The Jewish Journal, [en ligne], http://www.jewishjournal.com/hollywoodjew/item/fish_out_of_water _an_evening_with_arab-israeli_writer_sayed_kashua_20120427.

- Bhabha H. K. (2007). Les lieux de la Culture une théorie postcoloniale, Paris, Payot, 411 p.

- Bordas E. \& all. (2009). L'analyse littéraire, Paris, A. Colin, 256 p.

- Deleuze G. \& Guattari F. (1975). Kafka : Pour une littérature mineure, Paris, Éditions de Minuit, 159 p.

- Deleuze G. \& Parnet C. (1996). Dialogues, Paris, Flammarion, 187 p.

- Evrard F. (1997). La nouvelle, Paris, Seuil, 62 p.

- Gasquet A. \& Suãrez M. (2007). Écrivains multilingues et écritures métisses l'hospitalité des langues, Clermont-Ferrand, Presses Universitaires Blaise Pascal, 357 p.

- Habibi É. (1987). Les aventures extraordinaires de Saïd le Peptimiste, traduit de l'arabe par Jean-Patrick Guillaume, Paris, Gallimard, 208 p.

- Halutz D. (2010). «Language is my anchor», Haaretz, [en ligne] http://www.haaretz.com/weekend/magazine/language-is-my-anchor1.284042 .

- Hever H. (2001). Producing the Modern Hebrew Canon: Nation Building and Minority Discourse (New Perspectives on Jewish Studies), New-York, NYU Press, 280 p.

- Kamin D. (2013). «L'écrivain hébraïque contemporain le plus important est arabe », al-Masdar, [en ligne] http://www.al- masdar.net (en arabe).

- Kashua S. (2005). Cendrilla, Haaretz.

- Kashua S. (2006). Les Arabes dansent aussi, traduit de l'hébreu par Catherine Werchowsci, Paris, Éditions 10-18, 251 p.

- Kashua S. (2008-2012). עבודה ערבית (Travail arabe), Saisons 1, 2 \& 3 (hébreu - arabe).

- Kashua S. (2008). Et il y eut un matin, traduit de l'hébreu par Sylvie Cohen et Edna Degon, Paris, Points, 280 p.

- Kashua S. (2011). "A lesson in Arabic », Haaretz, [en ligne], http://www.haaretz.com/weekend/magazine/a-lesson-in-arabic1.348585 . 
- Kashua S. (2012). Deuxième personne, traduit de l'hébreu par Jean-Luc Allouche, Paris, Éditions de l'Olivier, 355 p.

- Kashua S. (2012). עבודה ערבית Saison 3, épisode 1, (hébreu - arabe).

- Kashua S. (2014). זהמר (c'est fini!), Haaretz, [en ligne], http://www.haaretz.co.il/magazine/sayed/.premium-1.2366140 (en hébreu).

- Kellman S. G. (1991). «Translingualism and the Literary Imagination », Criticism, Vol. 33, No. 4, 527-541.

- Kellman S.G. (2000). The Translingual Imagination, Nebraska Press, $160 \mathrm{p}$.

- Kraenker S. (2009). « Des écrivains à l'identité hybride, représentants d'une littérature-monde d'aujourd'hui et de demain : Karin Bernfeld, Nina Bouraoui, Assia Djébar, Amin Maalouf, Wajdi Mouawad », Synergies, Pays riverains de la Baltique, ${ }^{\circ} 6,219-227$.

- Lital L. (2014). Poetic Trespass : writing between Hebrew and Arabic in Israel/Palestine, Princeton University Press, 2014, xiii 337 p.

- Mansour A. (1969). In a New Light, traduit de l'hébreu par A. Birman, Vallentine Mitchell \& Co Ltd, $176 \mathrm{p}$.

- Mercier-Leca F. (2003). L'ironie, Paris, Hachette, 114 p.

- Neslen A. (2011). « Sayed Kashua, 33 : Journalist, Author, Scriptwriter, West Jerusalem Israel », dans In Your Eyes a Sandstorm : Ways of Being Palestinian, University of California Press, 101- 104.

- Propp V. (1970). Morphologie du conte, Paris, Seuil, 1970, 254 p.

- Reuter Y. (2005). L'analyse du Récit, Paris, A. Colin, Paris, 128 p.

- Schoentjes P. (2001). Poétique de l'ironie, Paris, Seuil, 347 p.

- Shammas A. (1988). Arabesques, traduit de l'hébreu par Guy Séniak, Arles, Actes Sud, 310 p.

- Shimony B. (2013). " Shaping Israeli-Arab Identity in Hebrew Words The Case of Sayed Kashua », Israel Studies, vol. 18, n 1, 146-169.

- Suleiman, Y. (2013). Arabic in the Fray, Language Ideology and Cultural Politics, Edinburgh University Press, 320 p.

- Torstrick R. (2000). The Limits of Coexistence, Identity Politics in Israel, The University of Michigan Press, $296 \mathrm{p}$.

- Todorov T. (1970). Introduction à la littérature fantastique, Paris, Seuil, $188 \mathrm{p}$.

- Valtette B. (1992). Le roman - initiation aux méthodes et aux techniques modernes d'analyse littéraire, Paris, Nathan, 128 p.

- Van Gorp H. \& all. (2005). Dictionnaire des termes littéraires, Paris, Champion classiques, 533 p. 
- Vilain P. (2009). L'autofiction en théorie, Chatou, Éditions de la Transparence, $123 \mathrm{p}$.

- Yanoshevsky G. (2011). «L'imposture comme posture : Sayed Kashua chroniqueur et romancier », dans Interférences littéraires/Literaire interferenties, nouvelle série, $\mathrm{n}^{\circ} 6$, «Postures journalistiques et littéraires », s. dir. Laurence van Nuijs, 151-168.

- Zimbalist D. פחדן מילדות -סייד קשוע (2009)(S. Kashua - Forever scared), film- documenatire, Heymann Brothers film. 